\title{
Nouvelles membranes minérales d'ultrafiltration : application à la purification de l'eau
}

\author{
par \\ C. GUIZARD*, J. A. ALARY*, A. LARBOT*, L. COT*, \\ M. RUMEAU**, B. CASTELAS** et J. GILLOT***
}

\section{R és u m é}

Les membranes réalisées offrent une gamme de porosité en ultrafiltration depuis un diamètre moyen de pore de $3 \mathrm{~nm}$ jusqu'à $10^{2} \mathrm{~nm}$. Constituées à base d'alumine ces membranes sont déposées sur la face interne d'un tube en alumine frittée. Elles ont une épaisseur comprise entre 1 et $5 \mu$.

L'ensemble support céramique plus membrane a été mis en œuvre sur un pilote d'ultrafiltration avec pour objectif la production d'eau potable à partir d'eaux douces polluées. Les essais effectués ont montré la possibilité d'obtenir une eau stérile sur une membrane de $3 \mu$ d'épaisseur et présentant des pores d'un diamètre moyen de $6 \mathrm{~nm}$; la perméabilité de la membrane, maintenue sur une durée de $1000 \mathrm{~h}$ étant dans ce cas de $5 \mathrm{l} / \mathrm{h} / \mathrm{m}^{2} / \mathrm{bar}$.

\section{S u $\mathrm{m} \mathbf{m}$ a r y}

New mineral barriers were prepared by depositing a thin film (1 to $5 \mu$ ) of alumina on the inner part of sintered alumina tubes. These membranes exhibit a porosity range from mean diameters of $3 \mathrm{~nm}$ to $10^{2} \mathrm{~nm}$. In order to optimize an ultrafiltration process designed to sterilize polluted fresh waters, pilot experiments were performed

\footnotetext{
* Laboratoire de chimie des matériaux (C.N.R.S. LA 314), Ecole Nationale Supérieure de Chimie, 8, rue de 1'Ecole Normale - 34075 Montpellier cedex.

** Laboratoire de Génie chimique et traitement des eaux, Université des Sciences et Techniques du Languedoc, place Eugène-Bataillon - 34060 Montpellier Cedex.

*** CERAVER, B.P. 113 - 65001 Tarbes.
} 
with sewage samples. Sterile water was obtained through a $3 \mu$ thick membrane displaying $6 \mathrm{~nm}$ diameter sized pores with a permeability of $5 \mathrm{l} / \mathrm{m}^{2} / \mathrm{h} /$ bar. The parameters of the final ultrafiltration process were tested over a thousand hours period without altering the permeation flow and the sanitary specifications of filtered water.

\section{INTRODUCTION}

Plusieurs enquêtes, menées au niveau mondial, prévoient une progression d'un facteur dix d'ici 1990, pour le marché de l'ultrafiltration. Il s'agit là de la plus forte croissance parmi les autres technologies à membranes (osmose inverse, microfiltration, électrodialyse, ...). Sur le plan scientifique et technique, cela signifie que les travaux poursuivis dans ce domaine au cours des 10 dernières années ont conduit à des membranes d'ultrafiltration dont la spécificité visà-vis des substrats à traiter s'est considérablement élargie. Bien que ne remettant pas en cause la place déjà occupée par les membranes organiques, les membranes minérales, de par leurs performances, devraient prendre une place de plus en plus importante dans ce secteur. En effet, les qualités intrinsèques des membranes minérales (résistance élevée à la pression, à la température et aux agents chimiques) permettent déjà d'accroître l'efficacité de certains procédés industriels et ouvrent de nouvelles possibilités d'utilisation par rapport aux membranes organiques.

Le travail présenté dans cet exposé concerne un nouveau type de membrane minérale à base d'alumine, mis au point dans notre laboratoire, et son application à la production d'eau potable. L'efficacité des procédés à membranes est lié au contrôle de trois types de paramètres qui influencent directement le phénomène de perméation sélective :

- paramètres chimiques : nature du substrat à traiter et interactions avec la membrane ; des pores;

- paramètres physiques : épaisseur de la membrane et diamètre

- paramètres dynamiques : pression et vitesse de circulation du substrat.

Lors de cette étude, les paramètres chimiques étant fixés par la nature de la couche en alumine, nous avons étudié les paramètres physiques et dynamiques liés à la stérilisation d'eaux douces polluées. Les conditions de préparation des membranes nous ont permis de réaliser une gamme de porosité couvrant le domaine de l'ultrafiltration depuis un diamètre moyen de pore de $3 \mathrm{~nm}$ jusqu'à $10^{2} \mathrm{~nm}$. Ce 
point est important dans la mesure où nous avons pu adapter la membrane au problème posé et non l'inverse.

\section{MATERIEL ET METHODES UTILISES}

Les membranes en alumine d'épaisseur 1 à $5 \mu$, ont été déposées sur la face interne de supports en alumine frittée de forme tubulaire, fournis par la Société Ceraver. Ces tubes d'un diamètre intérieur de $15 \mathrm{~mm}$, et d'un diamètre extérieur de $19 \mathrm{~mm}$, sont disponibles en $250 \mathrm{~mm}$ et $750 \mathrm{~mm}$ de longueur. Ces deux longueurs ont été utilisées pour nos expériences.

Ces membranes ont été mises en œuvre sur un pilote d'ultrafiltration (cf. fig. 1), comprenant :

- une boucle de recirculation du substrat dans laquelle est inséré le carter contenant la membrane. Cette boucle est alimentée par une pompe de circulation destinée à établir une vitesse de circulation comprise entre 0 et $5 \mathrm{~m} / \mathrm{s}$;

- une pompe d'alimentation destinée à compenser le débit de perméat, et maintenir la pression dans la boucle entre 0 et 30 bars.

Les membranes utilisées ont été caractérisées par microscopie électronique à balayage, sur un appareil Cambridge S 100 , en ce qui concerne leur morphologie, et par porosimétrie à mercure pour la détermination du volume poreux et du rayon des pores.

Le porosimètre à mercure utilisé est formé d'un circuit haute pression et d'un pénétromètre à mercure dans lequel on introduit l'échantillon. Ce pénétromètre comprend un capillaire calibré et un système d'électrodes permettant de mesurer la capacité entre le mercure et l'électrode externe. Au fur et à mesure que la pression augmente, le mercure pénètre dans les pores de plus en plus petits entrấnant une diminution de la capacité. La mesure effectuée donne donc la variation de la capacité en fonction de la pression. Les relations (1) et (2) suivantes permettent de calculer le rayon des pores ainsi que le volume poreux :

$$
\text { p.r }=-2 \tau \cos \theta(1)
$$

formule dans laquelle si $\mathrm{p}$ est exprimé en bar et $\mathrm{r}$ en $\mathrm{nm},-2 \tau \cos \theta$ est exprimé sous forme d'une constante égale à 7500 pour l'alumine avec $\tau=$ tension superficielle du mercure et $\theta$ l'angle de contact du mercure avec l'alumine :

$$
\mathrm{Vp}=\mathrm{K} \cdot \frac{\mathrm{\Sigma} \Delta \mathrm{C}}{\mathrm{m}}(2)
$$

le volume poreux ainsi exprimé est calculé en $\mathrm{mm}^{3} / \mathrm{g}$ pour $\Delta \mathrm{C}$ (variation de la capacité) exprimé en picofarad et $m$ (masse de l'échantillon) exprimé en gramme, $\mathrm{K}=10,18$. 


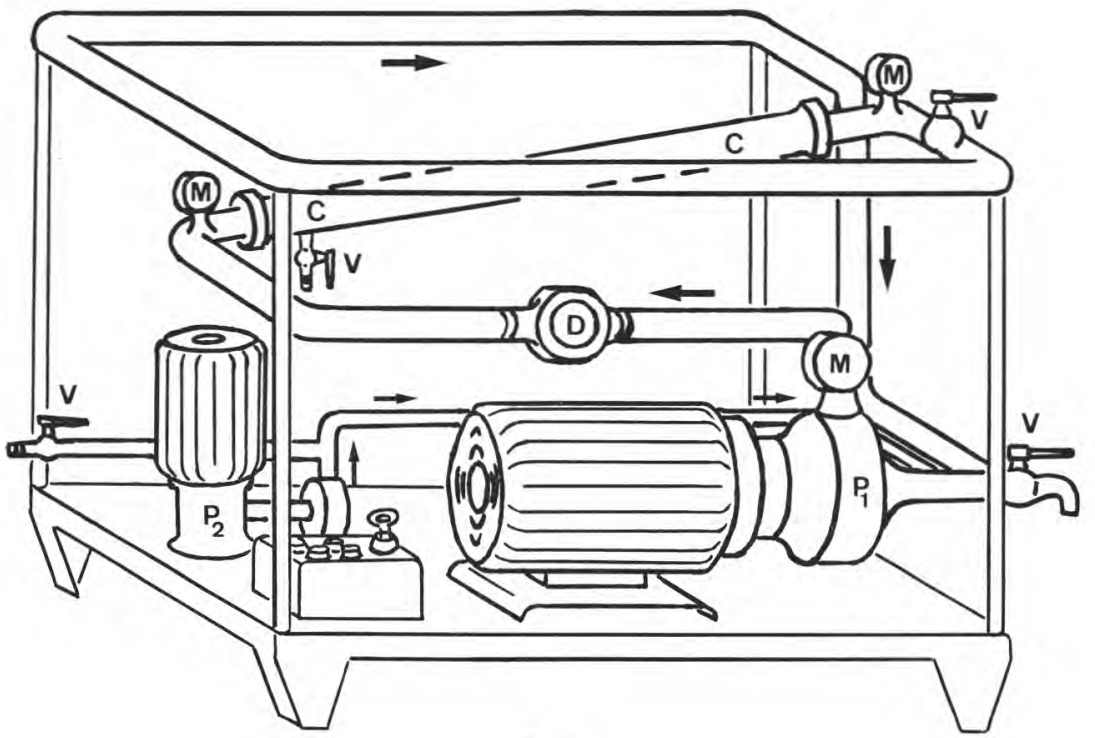

fig. 1

Pilote d'ultrafiltration destiné au traitement de l'eau. (C) : carter de réception du perméat entourant la membrane; (D) : débimètre destiné à mesurer la vitesse de circulation du substrat ; (M) : manomètres de contrôle de la pression de travail et de perte de charge ; (P1) : pompe doseuse d'alimentation ; (P2) : pompe de circulation; (V) : vannes de purge et de contrôle du débit.

Sur les eaux traitées, nous avons procédé à des analyses physicochimiques et bactériologiques avant et après ultrafiltration.

\section{RESULTATS}

Le support céramique utilisé (cf. fig. 2) présente une structure asymétrique obtenue par une juxtaposition de trois couches d'alumine poreuse dont l'épaisseur et le diamètre des pores sont successivement de $2 \mathrm{~mm}$ et $10 \mu, 40 \mu$ et $0,7 \mu, 15 \mu$ et $0,2 \mu$ 。

La couche d'alumine déposée sur ces supports constitue la membrane proprement dite, assurant la perméation sélective des espèces en présence dans le substrat. La figure 3 montre une image obtenue en microscopie électronique à balayage, sur une couche présentant un diamètre moyen des pores de $6 \mathrm{~nm}$. L'épaisseur de la couche est dans ce cas de $3 \mu$. Le diagramme, figure 4 , représente la courbe correspondante de répartition de la porosité, dans le support et dans la membrane. Dans ce cas la taille des pores mesurée dans la mem- 


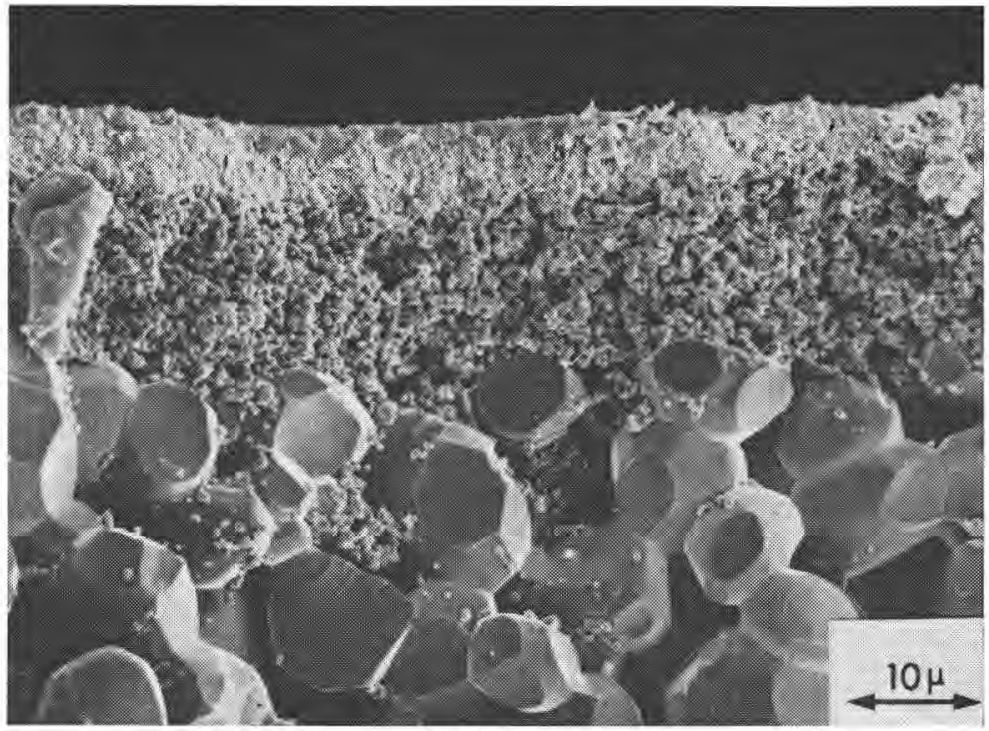

fig. 2

Image enregistrée au microscope électronique à balayage, d'une coupe transversale d'un tube CERAVER en alumine; grossissement X 1200.

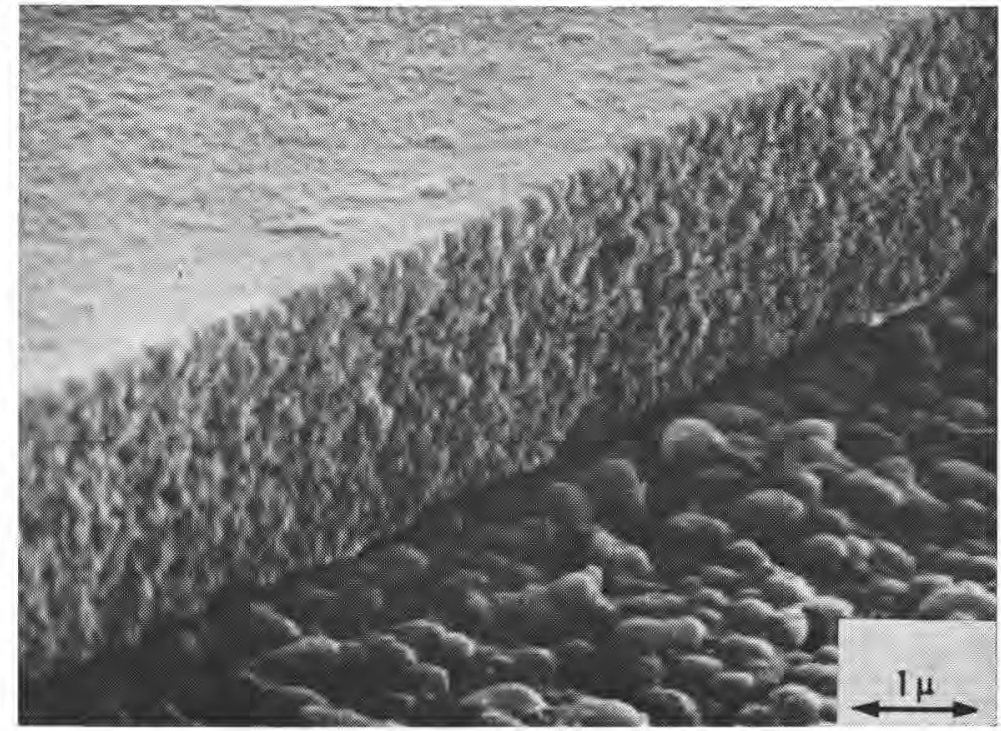

fig. 3

Image enregistrée au microscope électronique à balayage, d'une membrane en alumine déposée sur la surface interne d'un tube CERAVER ; grossissement X 12200 . 
brane constitue une limite inférieure pour l'appareil dont nous disposons. Cette limite est imposée par la pression maximum (5 000 bars), que l'on peut appliquer au mercure, afin de faire pénétrer celui-ci dans les pores de l'échantillon analysé. Comme le montrent les différents paliers sur la courbe, figure 4, la distribution de la taille des pores dans la membrane et dans le support reste très étroite. Au niveau de la membrane active une telle distribution assure une très grande sélectivité en ce qui concerne le seuil de coupure.

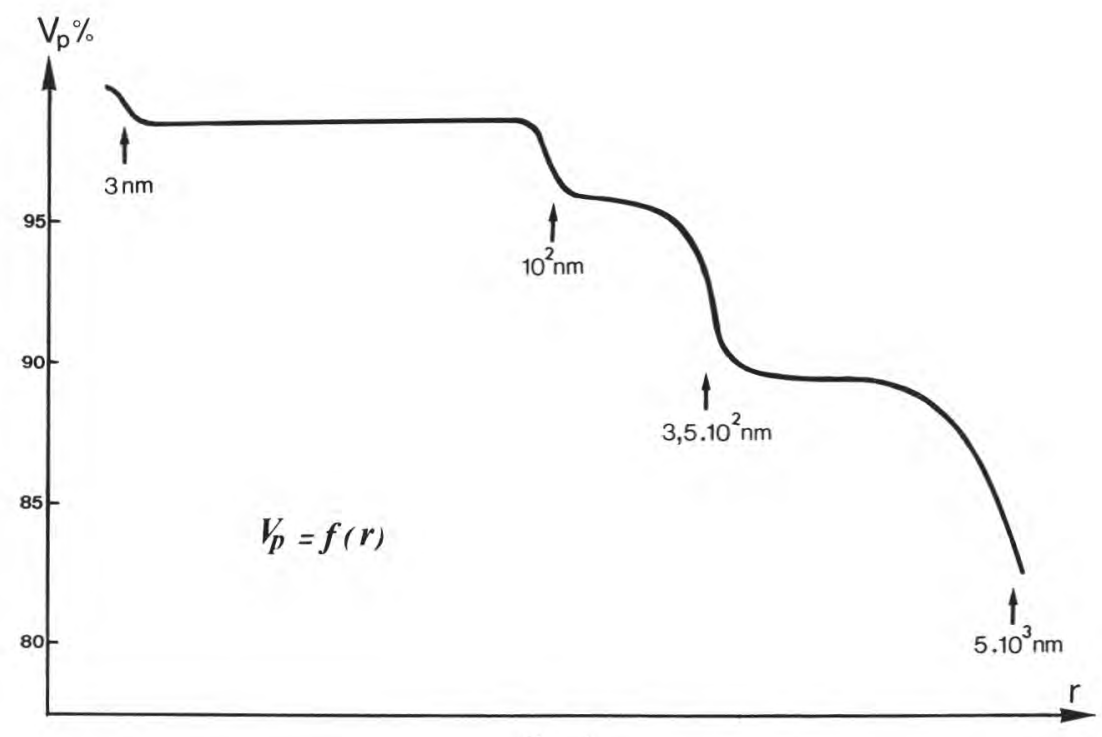

fig. 4

Courbe obtenue par porosimétrie à mercure, montrant la variation $V_{p}=f(r)$ du volume poreux en fonction du rayon des pores sur une membrane en alumine déposée sur un tube CERAVER.

Un des problèmes que nous nous sommes proposés de résoudre avec ce type de membrane, est celui de la production d'eau potable à partir d'eaux douces polluées. Plusieurs types d'eau (eau de rivière, de lac, de lagunage) ont été ultrafiltrés et analysés. Les conditions de rendement optimum du procédé ont été déterminées sur le site même d'une station de lagunage, permettant ainsi de disposer de tous les types d'eaux aux différents stades de leur épuration naturelle. Le procédé d'ultrafiltration mis au point utilise actuellement la membrane en alumine décrite ci-dessus de $3 \mu$ d'épaisseur dont le diamètre moyen des pores est de $6 \mathrm{~nm}$. Les paramètres dynamiques du système ont été fixés à $5 \mathrm{~m} / \mathrm{s}$ pour la circulation du substrat et 20 bars pour la 
pression de travail de la membrane. Ces conditions donnent lieu à l'établissement rapide d'un débit moyen de $100 \mathrm{l} / \mathrm{h} / \mathrm{m}^{2}$ pour une qualité d'eau dont les caractéristiques sont présentées dans le tableau 1. Ces résultats proviennent d'analyses physico-chimiques et bactériologiques effectuées sur une eau de lagunage avant et après traitement.

\section{TABLEAU 1}

Résultats des analyses bactériologiques et physico-chimiques sur une eau de lagunage avant et après ultrafiltration stérilisante

\begin{tabular}{l|c|c}
\hline & Eau brute & Eau ultrafiltrée \\
\hline Paramètres physico-chimiques & & \\
DCO $\left(\mathrm{O}_{2}\right.$ en mg/1) & $100-200$ & $0-10$ \\
Oxydabilité au $\mathrm{KMnO}_{4}$ & $1,12-1,30$ & $0,3-0,5$ \\
$\left(\mathrm{O}_{2}\right.$ en $\left.\mathrm{mg} / \mathrm{l}\right)$ & $51^{\circ}$ & $46^{\circ}$ \\
$\mathrm{TH}$ total (do français) & 118 & 1,5 \\
$\mathrm{SO}_{4}=(\mathrm{mg} / \mathrm{l})$ & 600 & 530 \\
$\mathrm{Cl}^{-}(\mathrm{mg} / \mathrm{l})$ & $9-21$ & $7-19$ \\
$\mathrm{NH}_{4}+(\mathrm{mg} / \mathrm{l})$ & $0,31-3,45$ & $0,06-0,09$ \\
$\mathrm{NO}_{2}^{-}(\mathrm{mg} / \mathrm{l})$ & 0,08 & - \\
$\mathrm{NO}_{3}^{-}(\mathrm{N}$ en mg/l) & &
\end{tabular}

Paramètres bactériologiques (volume des échantillons analysés : $100 \mathrm{ml}$ )

Bactéries hétérotrophes aérobies

Coliformes totaux

Coliformes fécaux

Incomptable
Incomptable
Incomptable

0

\section{DISCUSSION ET CONCLUSIONS}

Les essais d'épuration effectués sur une eau de lagunage nous ont montré qu'il était essentiel de pouvoir contrôler parfaitement le seuil de coupure de la membrane, afin d'éviter un colmatage interne de la couche filtrante. Ce seuil de coupure est déterminé par la taille des colloïdes présents dans le milieu. Ils représentent une limite supé- 
rieure pour la porosité, au-dessus de laquelle il se produit un colmatage irréversible de la membrane, conduisant rapidement à une chute importante du débit de perméation. Un diamètre de pore de $6 \mathrm{~nm}$, semble être dans ce cas une dimension optimum pour assurer un débit de perméation élevé en évitant le colmatage interne de la membrane. Les analyses bactériologiques présentées dans le tableau 1, montrent clairement que cette taille de pore est largement suffisante pour assurer une stérilisation complète de l'eau. En ce qui concerne les analyses physico-chimiques, on note une diminution significative des sulfates, nitrates et nitrites dans les eaux traitées, alors que les ions chlorure et ammonium ne sont pas arrêtés. Cette rétention sélective de certains ions peut s'expliquer par une augmentation de taille due à une complexation avec certains éléments présents dans l'eau de départ. A ce sujet, on a constaté que plus l'eau de départ était chargée en matières organiques, meilleure était la rétention des ions sulfates, nitrates et nitrites. Par contre, les ions chlorure et ammonium non complexés migrent normalement à travers la membrane, quelle que soit la nature et la composition de l'eau de départ.

A partir d'un seuil de coupure correctement ajusté, les conditions d'écoulement tangentiel du fluide le long de la membrane influent directement sur le débit de perméation. Afin de réduire au maximum la formation de la couche de polarisation provoquant le plus souvent un colmatage de surface, il est important soit d'établir un régime d'écoulement turbulent au voisinage de la membrane, soit d'opérer un décolmatage à intervalles réguliers de la membrane. Un rendement de perméation élevé a été obtenu en optimisant les valeurs de travail des paramètres pression et circulation dans la boucle d'ultrafiltration. En effet, la valeur moyenne du débit de perméation est directement reliée au transfert de masse à travers la membrane qui consiste en :

- diffusion-migration dans la couche de polarisation ;

- adsorption par la membrane ;

- diffusion dans la membrane.

On peut mettre en évidence l'évolution de la couche de polarisation, en traçant la courbe donnant le débit en fonction de la pression (cf. fig. 5a). Cette courbe présente deux parties : une dans laquelle le débit augmente régulièrement avec la pression, l'autre où le débit atteint très rapidement une valeur limite. D'autre part, si l'on trace la courbe donnant le profil de concentration dans le substrat, il apparaît deux zones successives avec à l'interface, un gradient de concentration important ; la concentration la plus élevée étant au voisinage de la membrane dans la couche de polarisation d'épaisseur $\Delta x$. Dans ce cas, on peut émettre l'hypothèse que deux phénomènes se superposent (cf. fig. $5 \mathrm{~b}$ ) ; le premier de convection assurant un flux de matière du substrat vers la couche de polarisation et la membrane; le deuxième de diffusion du milieu le plus concentré, vers le milieu le moins concentré, c'est-à-dire de la couche de polarisa- 
tion vers le substrat. Ce phénomène de diffusion sera d'autant plus important que le gradient de concentration entre la couche et le substrat sera élevé. Le profil de la courbe $\mathrm{D}=\mathrm{f}(\mathrm{P})$, dans la zone 1 , s'explique par un phénomène de convection prépondérant, qui augmente en même temps que la pression P. Dans la zone 2, la convection augmente avec la pression, mais le flux de matière correspondant est compensé par la diffusion opérant en sens inverse, jusqu'à l'établissement d'un équilibre donnant le débit maximum de la membrane. Une deuxième hypothèse consiste, tout simplement, à envisager la formation d'un gel, à partir d'une solution saturée, due à la couche de polarisation ; la densité de ce gel influant directement sur le débit de perméation.

Dans les deux cas, il est clair que les performances de la membrane sont directement liées à la vitesse de formation de la couche de polarisation et au gradient de concentration à l'interface, avec le substrat. Un certain nombre de solutions sont envisageables, pour atténuer ce phénomène de polarisation. On peut citer par exemple :

- la diminution de la viscosité du substrat, par élévation de la température ;

- l'augmentation de la vitesse de circulation, afin d'établir un régime turbulent à proximité de la membrane ;

- introduire des promoteurs de turbulence dans la boucle d'ultrafiltration ;

- procéder à un décolmatage périodique de la membrane, par pression inverse.

Cette dernière solution est un avantage important des membranes minérales, par rapport aux membranes organiques.

Dans le cas de l'eau, nous avons déterminé des valeurs pour les paramètres dynamiques, pression et vitesse de circulation, pour lesquels la perméabilité de la membrane est de $10 \mathrm{l} / \mathrm{h} / \mathrm{m}^{2} / \mathrm{bar}$. Cette perméabilité diminue rapidement dans la première heure de mise en service de la membrane pour atteindre une perméabilité moyenne de $5 \mathrm{l} / \mathrm{h} / \mathrm{m}^{2} /$ bar. Ce dernier chiffre a été maintenu pour une durée de $1000 \mathrm{~h}$, avec un décolmatage périodique par pression inverse dont les séquences sont programmées toutes les $4 \mathrm{~h}$. La qualité de l'eau ultrafiltrée est restée constante au cours de cette période.

En conclusion, les résultats de cette étude sur l'eau ont montré qu'il était indispensable de maîtriser l'ensemble des paramètres physiques, chimiques et dynamiques liés au fonctionnement de la membrane pour aboutir à un procédé performant. Ce n'est donc pas de la seule amélioration des procédés que dépendent les performances des membranes, mais également des modifications à apporter à leur composition chimique et à leur état de surface, souvent responsables d'interactions parasites entre le substrat et la membrane. C'est dans 

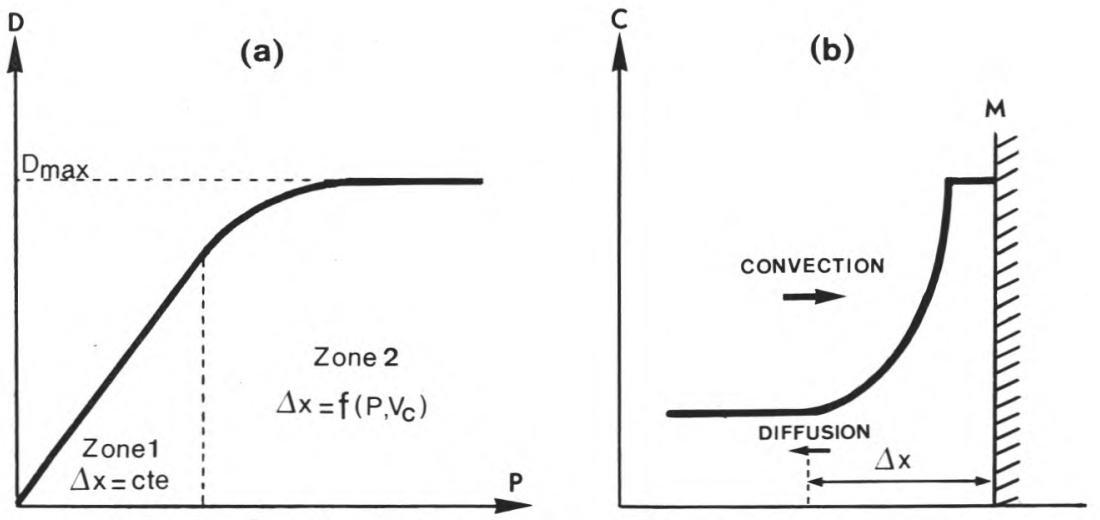

fig. 5

a) Courbe $\mathrm{D}=\mathrm{f}(\mathrm{P})$ donnant le débit de perméat en fonction de la pression appliquée.

b) Profil de concentration dans le substrat au voisinage de la membrane.

ce but que nous poursuivons actuellement des études fondamentales en chimie des solides, conduisant à l'élaboration de matériaux nouveaux, dont d'aspect essentiel est la mise en évidence de relations entre structure et propriétés dans les couches minérales minces à porosité contrôlée. Nous pensons ainsi élargir les possibilités d'utilisation de ces membranes, en améliorant leur spécificité vis-à-vis des substrats à traiter.

\section{Remerciements}

Nous remercions l'ANVAR Languedoc-Roussillon ainsi que les sociétés CERAVER et IMECA pour l'aide financière qu'elles ont apportée à cette étude. 